\title{
Cambios interanuales en la biomasa y biodiversidad de zooplancton gelatinoso en aguas interiores de Chiloé, sur de Chile (primaveras 2004 y 2005)
}

\author{
Interannual changes in biomass and diversity of the gelatinous zooplankton \\ in the interior waters of Chiloé, southern Chile (Springs 2004 and 2005) \\ Francisco Villenas ${ }^{1}$, Daniela Soto $^{1}$ y Sergio Palma ${ }^{1}$ \\ ${ }^{1}$ Escuela de Ciencias del Mar, Pontificia Universidad Católica de Valparaíso, Casilla 1020, Valparaíso, Chile \\ spalma@ucv.cl
}

\begin{abstract}
Zooplankton biomass was compared between the fraction of chitinous and gelatinous organisms from the southern region of Chile between Puerto Montt and Boca del Guafo, in the springs of 2004 and 2005. The biomass values were higher in 2004, being mainly composed by chitinous fraction, particularly in the north microbasin. Conversely, during 2005 , a sharp increase of the gelatinous fraction was observed reaching $60 \%$ of the total biomass in the northern microbasin, and gradually decreasing into the Boca del Guafo. The increase in biomass seemed to be a result of the explosive proliferation of the siphonophore Muggiaea atlantica, whose densities increased 5.6 folds in 2005, triggering a drastic decrease in diversity of the gelatinous zooplankters. In both springs, 34 species of semi- and gelatinous organisms were identified, distributed in six chaetognaths, six siphonophores and 22 hydromedusae. Chaetognaths and siphonophores had previously been registered for the study area, but all hydromedusae encompass new records for the area between Puerto Montt and Boca del Guafo.
\end{abstract}

Key words: Zooplankton biomass, chaetognaths, siphonophores, hydromedusae, Chiloé Island

\section{Introducción}

El sistema de canales y fiordos chilenos es uno de los sistemas estuarinos más grandes del mundo, con una extensión aproximada de 1.600 km de longitud y un área de alrededor de $240.000 \mathrm{~km}^{2}$ (Palma \& Silva 2004). Este ecosistema es único por su elevada complejidad hidrográfica y geomorfológica, unida a su alta variabilidad climática. Este ecosistema se forma como resultado de la acción erosiva de glaciares y el hundimiento tectónico del valle longitudinal al sur de Puerto Montt (Borgel 1970-71). Se caracteriza por una circulación estuarina de dos capas, con un flujo de salida en superficie y uno de entrada en profundidad; diferentes grados de mezcla vertical influenciados por la
Resumen.- Se comparó la biomasa zooplanctónica entre organismos quitinosos y gelatinosos en la región austral de Chile, entre Puerto Montt y la Boca del Guafo, en las primaveras de 2004 y 2005. Los valores de biomasa fueron mayores en el 2004 y se mantuvieron sostenidos fundamentalmente por la fracción quitinosa, especialmente en la microcuenca norte. En el 2005 se determinó un fuerte incremento de la fracción gelatinosa que alcanzó el $60 \%$ en la microcuenca norte y disminuyó gradualmente hacia la Boca del Guafo. Este incremento se explica por la proliferación explosiva del sifonóforo Muggiaea atlantica, cuya densidad aumentó 5,6 veces en el 2005, causando una fuerte disminución en la diversidad de zooplancton gelatinoso. En ambas primaveras, se identificaron 34 especies de organismos semigelatinosos y gelatinosos, distribuidas en seis de quetognatos, seis de sifonóforos y 22 de hidromedusas. De este total, los quetognatos y sifonóforos habían sido previamente registrados para el área de estudio, pero la totalidad de las hidromedusas constituyen nuevos registros para el área entre Puerto Montt y Boca del Guafo.

Palabras clave: Biomasa zooplanctónica, quetognatos, sifonóforos, hidromedusas, isla de Chiloé geomorfología de la zona; condiciones oceanográficas y meteorológicas variables, y amplios rangos de marea (Silva et al. 1995, 1997). La microcuenca norte (seno de Reloncaví y golfo Ancud) se caracteriza por una mayor estabilidad de la columna de agua producto del agua estuarina que favorece la estratificación y una mayor productividad biológica. En cambio, la microcuenca sur (golfo Corcovado y Boca del Guafo) tiene una menor estabilidad vertical debido al ingreso permanente de agua subantártica y a la mayor mezcla causada por acción del viento y las mareas (Ramírez \& Pizarro 2005, Palma \& Rosales 1997, Palma \& Silva 2004).

Desde mediados de los años 80, este ecosistema se ha visto afectado por un incremento sostenido de 
actividades productivas, tanto en el ámbito turístico como acuícola, particularmente entre la isla de Chiloé y la región de Aysén. A nivel mundial, se ha observado una estrecha relación entre el aumento de las actividades antrópicas y la eutroficación, la introducción de nuevas especies y la sobrepesca (Jackson et al. 2001, Brodeur et al. 2002, Kidwell 2007). Por lo tanto, es importante conocer la biodiversidad planctónica pues hay cambios en la composición de especies que pueden afectar los flujos de materia y energía, así como las interacciones tróficas que favorecen la abundancia de las especies que controlan estos flujos (Chapin et al. 2000).

En términos generales, la biomasa zooplanctónica en los canales y fiordos australes chilenos está constituida principalmente por copépodos, quetognatos, eufáusidos y carnívoros gelatinosos (medusas, sifonóforos y ctenóforos) (Palma \& Silva 2004). En los últimos años, el estudio del macroplancton gelatinoso ha cobrado mayor relevancia por el impacto que provoca en la estructura comunitaria de diversas áreas marinas (Mills 2001, Pagès et al. 2001, Brodeur et al. 2002, Giesecke \& González 2004), principalmente por su capacidad para formar densas agregaciones y dominar estacionalmente la biomasa de zooplancton (Mianzan \& Guerrero 2000). Sin embargo, las estimaciones de biomasa de organismos gelatinosos son poco desarrolladas debido a los problemas metodológicos involucrados (Arai 1988), lo cual dificulta la comprensión de su papel ecológico en los procesos de transferencia energética en los sistemas marinos.

En el presente estudio se analizaron los cambios interanuales en la biomasa de zooplancton en aguas interiores de Chiloé, en las primaveras de 2004 y 2005. En ambos períodos se analizó el aporte de la fracción gelatinosa y quitinosa a la biomasa, así como la abundancia y biodiversidad del zooplancton gelatinoso, en relación con las condiciones oceanográficas existentes en esos periodos del año.

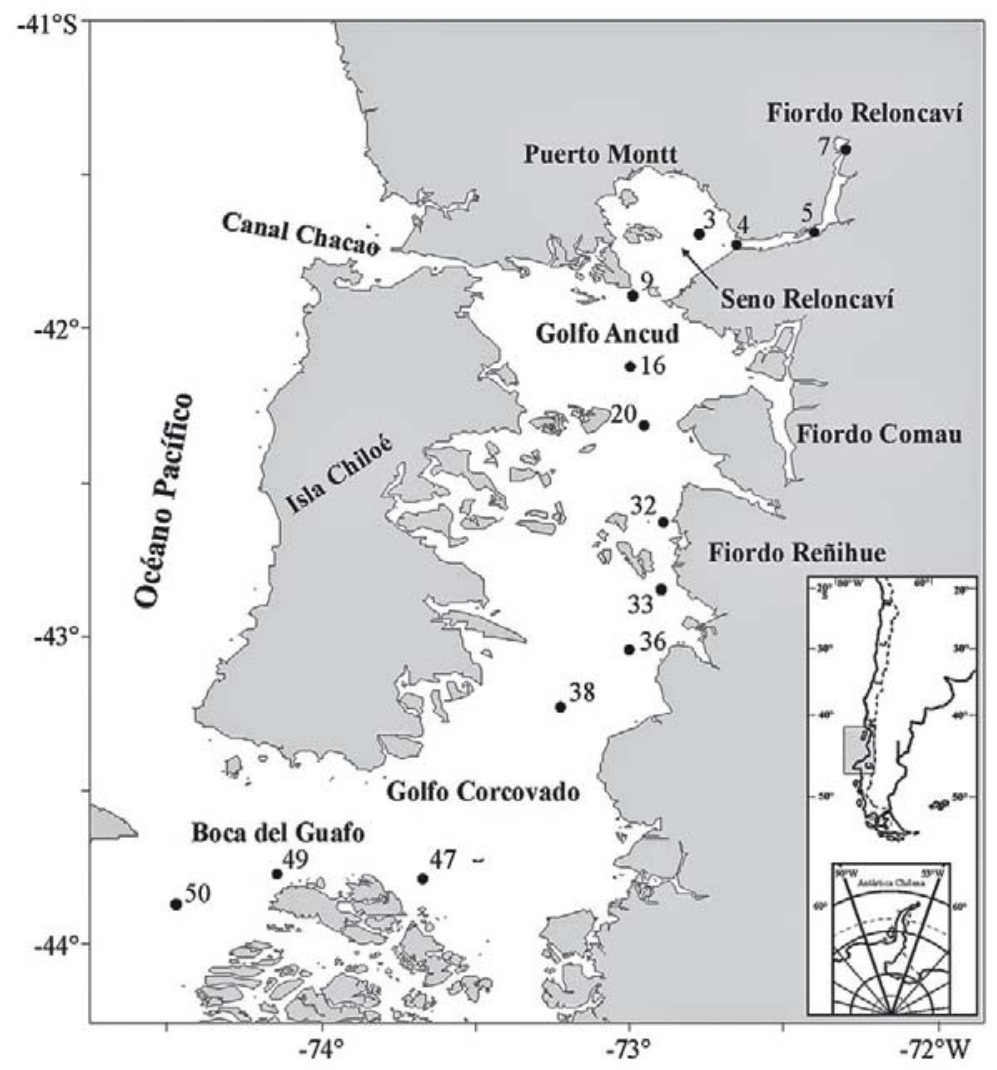

Figura 1

\section{Ubicación de las estaciones de muestreo zooplanctónico entre el fiordo de Reloncaví y la Boca del Guafo}




\section{Material y métodos}

Durante el desarrollo de los cruceros CIMAR 10 Fiordos (12-23 noviembre 2004) y CIMAR 11 Fiordos (7-27 noviembre 2005), se obtuvieron muestras de zooplancton en 14 estaciones oceanográficas distribuidas en un

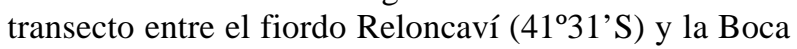
del Guafo (4339'S) (Fig. 1). En cada estación se efectuaron arrastres oblicuos de zooplancton desde un máximo de $200 \mathrm{~m}$ a superficie, utilizando redes Bongo de $350 \mu \mathrm{m}$ de abertura de mallas y 60 cm de diámetro de boca.

Las muestras se conservaron en agua de mar con formalina al 5\%, neutralizada con tetraborato de sodio para su posterior análisis. De cada muestra se determinó: a) la biomasa quitinosa constituida fundamentalmente por copépodos, eufáusidos y larvas de crustáceos, y b) la biomasa gelatinosa-semigelatinosa aportada por sifonóforos, medusas, ctenóforos y quetognatos. Las mediciones se realizaron mediante el método de desplazamiento de volumen y los resultados se expresaron en volumen de plancton húmedo por $1000 \mathrm{~m}^{3}$ (mL 1000 $\mathrm{m}^{-3}$ ). De cada muestra se separaron, contaron e identificaron quetognatos, sifonóforos y medusas, y además se calculó el índice de diversidad específica para estos organismos. Para describir los patrones de distribución geográfica, se consideraron solo las especies dominantes y su abundancia se estandarizó en número de individuos por $1000 \mathrm{~m}^{3}$ (ind. $1000 \mathrm{~m}^{-3}$ ).

En todas las estaciones oceanográficas se obtuvieron registros de temperatura $\left({ }^{\circ} \mathrm{C}\right)$, salinidad y concentración de oxígeno disuelto ( $\left.\mathrm{mL} \mathrm{L}^{-1}\right)$ con un CTD Seabird modelo 25 , instalado en una roseta con 24 botellas Niskin. Dado que los muestreos de zooplancton fueron oblicuos en la columna de agua (0-200 m), para cada estación oceanográfica se calcularon los valores promedio de temperatura, salinidad y oxígeno disuelto en la columna de agua definida.

Para determinar el grado de dependencia entre las variables abióticas y las especies dominantes, se aplicó el análisis de correlación de Pearson, para lo cual su abundancia se normalizó mediante la transformación ln $\left(x^{+1}\right)$ (Sokal \& Rohlf 1995). Además, se utilizó la prueba no paramétrica de Mann-Whitney para determinar si la abundancia de las especies dominantes fue significativamente diferente entre las primaveras de 2004 y 2005.

\section{Resultados}

\section{Condiciones oceanográficas}

En primavera de 2004, la temperatura promedio en la columna de agua fluctuó entre 9,9 y $11,4^{\circ} \mathrm{C}$. En la

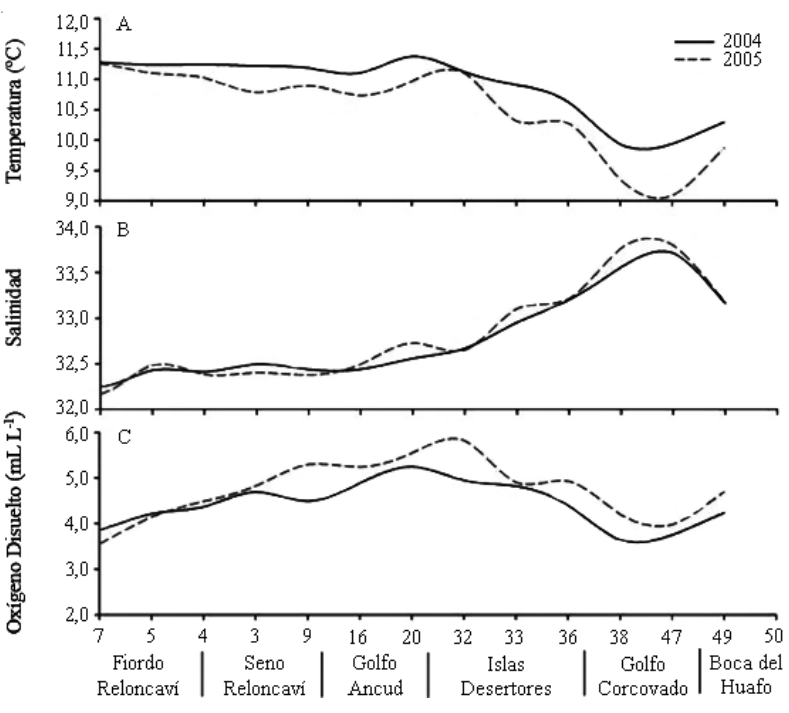

Figura 2

\author{
Distribución latitudinal de A) temperatura $\left.\left({ }^{\circ} \mathrm{C}\right), \mathrm{B}\right)$ \\ salinidad, y $\mathrm{C})$ oxígeno disuelto $\left(\mathrm{mL} \mathrm{L} \mathrm{L}^{-1}\right)$ \\ entre 0 y $200 \mathrm{~m}$ de profundidad \\ Latitudinal distribution of A) temperature $\left.\left({ }^{\circ} \mathrm{C}\right), \mathrm{B}\right)$ \\ salinity, and $\mathrm{C}$ ) dissolved oxygen $\left(\mathrm{mL} \mathrm{L}^{-1}\right)$, \\ between 0 and $200 \mathrm{~m}$ depth
}

microcuenca norte, los valores medios fueron más altos y muy similares entre sí, mientras que en la microcuenca sur, se registró un descenso gradual de temperatura desde las islas Desertores hasta la Boca del Guafo. En primavera de 2005 se determinó el mismo patrón de distribución latitudinal de temperatura promedio, pero los valores fueron menores $\left(9,1 \mathrm{y} 11,3^{\circ} \mathrm{C}\right)$, particularmente en el golfo Corcovado (Fig. 2a).

En ambas primaveras se observó el mismo patrón de distribución latitudinal de salinidad, con un incremento gradual desde el fiordo Reloncaví hasta el golfo de Corcovado, donde se registraron los mayores valores, y luego disminuyeron hacia la Boca del Guafo (Fig. 2b). Los valores medios de salinidad fluctuaron entre 32,2 y 33,8 en ambas primaveras, siendo ligeramente superiores en el 2005.

El patrón de distribución latitudinal de oxígeno disuelto fue muy similar en ambas primaveras, con máximos en el golfo de Ancud. Los valores promedio fluctuaron entre 3,6 y 5,2 $\mathrm{mL} \mathrm{L}^{-1}$ el 2004, y entre 3,0 y $5,8 \mathrm{~mL} \mathrm{~L}^{-1}$ el 2005, cuando fueron mayores (Fig. 2c). 


\section{Fracciones quitinosa y gelatinosa de la biomasa zooplanctónica}

En ambas primaveras la biomasa quitinosa estuvo dominada esencialmente por copépodos, eufáusidos y larvas de crustáceos. En primavera de 2004, los máximos de biomasa fueron un orden de magnitud mayor que el 2005 (Fig. 3). En el 2004 los valores fluctuaron entre 12 y $1.421 \mathrm{~mL} 1000 \mathrm{~m}^{-3}$, con una media de $681 \pm 623 \mathrm{~mL}$ $1000 \mathrm{~m}^{-3}$, siendo extremadamente altos en la microcuenca norte, particularmente en el golfo Ancud. En cambio el 2005, los valores fueron relativamente bajos, con menor coeficiente de variabilidad y fluctuaron entre 41 y 547 $\mathrm{mL} 1000 \mathrm{~m}^{-3}$, con un promedio de $215 \pm 161 \mathrm{~mL} 1000$ $\mathrm{m}^{-3}$, observándose un aumento de la biomasa quitinosa en la microcuenca sur (Fig. 3).

La biomasa gelatinosa fue sustentada principalmente por quetognatos, sifonóforos y medusas. En ambas primaveras se estimaron bajos valores de biomasa; para

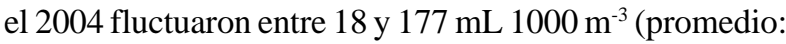

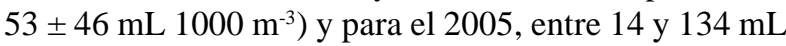
$1000 \mathrm{~m}^{-3}$ (promedio: $60 \pm 31 \mathrm{~mL} 1000 \mathrm{~m}^{-3}$ ). En primavera de 2004 fue notoria la disminución de la fracción

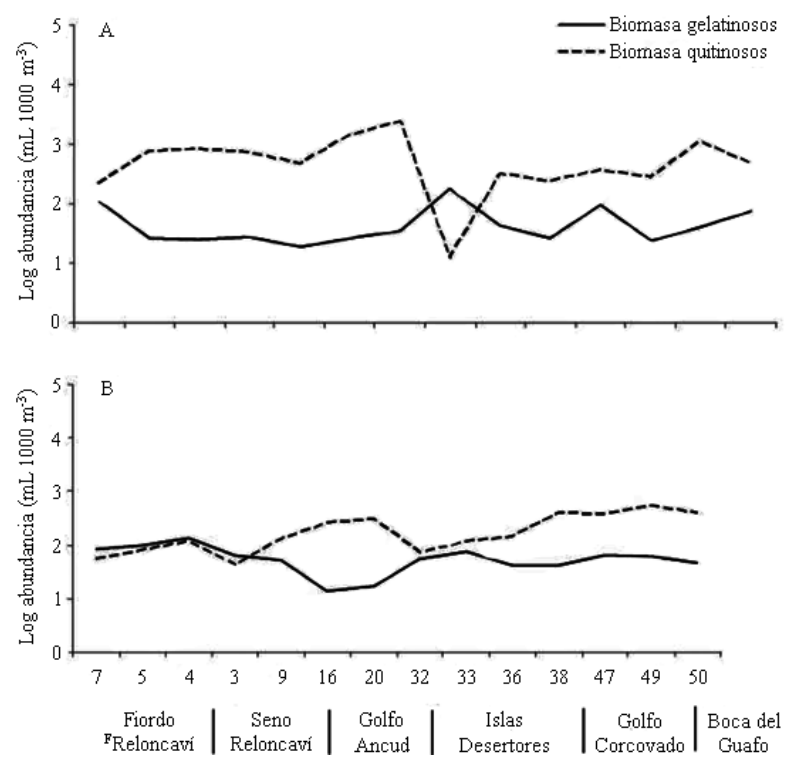

Figura 3

Distribución latitudinal de las fracciones quitinosa y gelatinosa de la biomasa zooplanctónica. A) primavera 2004, B) primavera 2005

Latitudinal distribution of the chitinous and gelatinous fractions of the zooplankton biomass. A) spring 2004, B) spring 2005 gelatinosa en la microcuenca norte, que estuvo asociada al fuerte incremento de la fracción quitinosa. En cambio en el 2005 se observó un fuerte incremento de la fracción gelatinosa en la microcuenca norte, asociado a una baja en la fracción quitinosa.

El porcentaje de contribución de las fracciones quitinosa y gelatinosa (Fig. 3) indicó que en primavera de 2004, los organismos gelatinosos fueron importantes solo en la estación 32, mientras que el 2005, se observó un mayor aporte de los gelatinosos a la biomasa total, alcanzando el $60 \%$ en la microcuenca norte, disminuyendo gradualmente de norte a sur (Fig. 3).

\section{Diversidad}

En ambas primaveras los valores de diversidad específica de los organismos gelatinosos fueron muy heterogéneos. Los mayores valores se obtuvieron en primavera de 2004 y fluctuaron entre 0,35 y 3,03 bits, mientras que el 2005 fluctuaron entre 0,29 y 2,14 bits. En ambos periodos se observaron altos valores en ambas microcuencas a excepción de las estaciones situadas en torno a las islas Desertores, donde se determinaron los menores valores de diversidad (Fig. 4).

\section{Composición específica}

En ambas primaveras, se identificaron 34 especies de organismos gelatinosos (27 el 2004 y 32 el 2005), distribuidas en seis especies de quetognatos, seis de sifonóforos y 22 de hidromedusas. Todas las especies de hidromedusas se registran por primera vez para el área entre el fiordo Reloncaví y la Boca del Guafo, pues

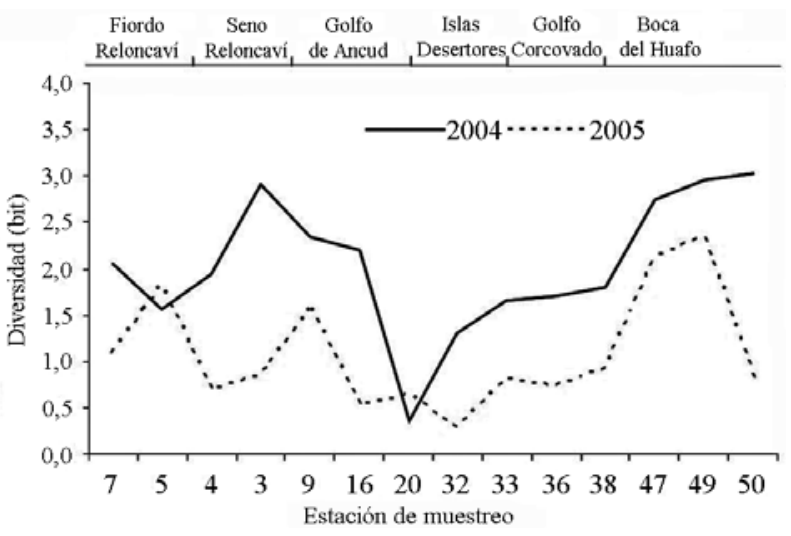

Figura 4

Índice de diversidad de zooplancton gelatinoso en las primaveras de 2004 y 2005

Diversity index of gelatinous zooplankton in 2004 and 2005 springs 
estudios previos de Galea (2007) y Galea et al. (2007), describen 12 especies que fueron recolectadas exclusivamente al interior del fiordo Comau (Tabla 1).

En primavera de 2004, las especies dominantes fueron los quetognatos Sagitta tasmanica (83,8\%) y Eukrohnia hamata (7,0\%), los sifonóforos Muggiaea atlantica (90,0\%) y Pyrostephos vanhoeffeni $(5,1 \%)$ y las hidromedusas Solmundella bitentaculata (27,9\%), Clytia simplex (21,7\%), Hydractinia tenuis (18,1\%), Amphogona apicata $(11,8 \%)$ y Bougainvillia muscoides $(7,2 \%)$ (Tabla $1)$. En la primavera de 2005, fueron los quetognatos Sagitta tasmanica (56,1\%), Eukrohnia hamata (11,8\%), Sagitta decipiens (16,9\%) y Sagitta chilensis (9,5\%), los sifonóforos $M$. atlantica $(98,6 \%)$ y las hidromedusas $S$. bitentaculata (25,5\%), C. simplex (18,4\%), Cunina peregrina (11,0\%) y Obelia spp. (9,5\%) (Tabla 1).

Tabla 1

Abundancia promedio, abundancia total, porcentaje de abundancia y frecuencia de ocurrencia de quetognatos, sifonóforos y medusas en la zona de estudio. En negrita se indican las especies dominantes en las primaveras de 2004 y 2005. Con un asterisco se indican las especies descritas previamente para el fiordo Comau (Galea 2007, Galea et al. 2007)

Chaetognaths, siphonophores and hydromedusae mean and total abundance, percentage of abundance and frequency of occurrence in the study area. Dominant species in spring 2004 and 2005 are indicated in bold. Species previously described from Comau Fjord (Galea 2007, Galea et al. 2007) are indicated with an asterisk

\begin{tabular}{|c|c|c|c|c|c|c|c|c|}
\hline \multirow[t]{2}{*}{ Especie } & \multicolumn{2}{|c|}{$\begin{array}{l}\text { Promedio } \\
\text { (ind } 1000 \mathrm{~m}^{-3} \text { ) }\end{array}$} & \multicolumn{2}{|c|}{$\begin{array}{c}\text { Total } \\
\text { (ind } 1000 \mathrm{~m}^{-3} \text { ) }\end{array}$} & \multicolumn{2}{|c|}{$\begin{array}{c}\text { Porcentaje } \\
(\%)\end{array}$} & \multicolumn{2}{|c|}{ Frecuencia } \\
\hline & 2004 & 2005 & 2004 & 2005 & 2004 & 2005 & 2004 & 2005 \\
\hline \multicolumn{9}{|l|}{ Chaetognatha } \\
\hline Sagitta tasmanica & 1764 & 718 & 26464 & 10045 & 83,8 & 56,1 & 100 & 92,9 \\
\hline Eukrohnia hamata & 147 & 151 & 2213 & 2116 & 7,0 & 11,8 & 40,0 & 35,7 \\
\hline Sagitta decipiens & 62 & 216 & 928 & 3029 & 2,9 & 16,9 & 46,6 & 35,7 \\
\hline Sagitta chilensis & 39 & 121 & 582 & 1693 & 1,8 & 9,5 & 26,6 & $\mathbf{5 0 , 0}$ \\
\hline Sagitta enflata & 57 & 33 & 850 & 457 & 2,7 & 2,6 & 60,0 & 21,4 \\
\hline Sagitta minima & 34 & 40 & 520 & 555 & 1,6 & 3,1 & 66,6 & 35,7 \\
\hline \multicolumn{9}{|l|}{ Siphonophorae } \\
\hline Muggiaea atlantica & 2857 & 17127 & 42852 & 239771 & 90,0 & 98,6 & 100 & 100 \\
\hline Pyrostephos vanhoeffeni & 162 & 139 & 2433 & 1939 & 5,1 & 0,8 & 40,0 & 64,3 \\
\hline Sphaeronectes gracilis & 108 & 75 & 1620 & 1044 & 3,4 & 0,4 & 66,7 & 71,4 \\
\hline Lensia conoidea & 259 & 30 & 259 & 416 & 0,5 & 0,2 & 26,7 & 35,7 \\
\hline Dimophyes arctica & 29 & 0 & 439 & 6 & 0,9 & 0 & 13,3 & 7,1 \\
\hline Sphaeronectes fragilis & 0 & 8 & 0 & 105 & 0 & 0 & 0,0 & 21,4 \\
\hline \multicolumn{9}{|l|}{ Hydromedusae } \\
\hline Solmundella bitentaculata* & 530 & 271 & 7950 & 3795 & 27,9 & 25,5 & 66,7 & 71,4 \\
\hline Clytia simplex* & 412 & 196 & 6177 & 2746 & 21,7 & 18,4 & 93,3 & 100 \\
\hline Hydractinia tenuis* & 343 & 5 & 5143 & 74 & 18,1 & 0,4 & 40,0 & 14,3 \\
\hline Amphogona apicata* & 223 & 4 & 3350 & 54 & 11,8 & 0,4 & 66,7 & 7,1 \\
\hline Bougainvillia muscoides* & 137 & 52 & 2062 & 730 & 7,2 & 4,9 & 86,7 & $\mathbf{5 7 , 1}$ \\
\hline Obelia spp.* & 44 & 101 & 654 & 1414 & 2,3 & 9,5 & 33,3 & 57,1 \\
\hline Cunina peregrina & 45 & 118 & 673 & 1650 & 2,4 & 10,0 & 33,3 & 35,7 \\
\hline Hybocodon unicus & 5 & 13 & 371 & 177 & 1,3 & 1,2 & 66,7 & 35,7 \\
\hline Hydractinia borealis* & 35 & 14 & 523 & 192 & 1,8 & 1,3 & 53,3 & 35,7 \\
\hline Proboscidactyla ornata & 32 & 6 & 484 & 82 & 1,7 & 0,5 & 60,0 & 14,3 \\
\hline Leuckartiara octona* & 29 & 14 & 430 & 202 & 1,5 & 1,0 & 66,7 & 42,9 \\
\hline Euphysa aurata* & 19 & 0 & 290 & 900 & 1,0 & 6,0 & 26,7 & 57,1 \\
\hline Ectopleura dumortieri* & 10 & 8 & 150 & 107 & 0,5 & 0,7 & 20,0 & 21,4 \\
\hline Proboscidactyla stellata* & 5 & 1 & 79 & 13 & 0,3 & 0,1 & 26,7 & 14,3 \\
\hline Rophalonema velatum & 4 & 8 & 65 & 106 & 0,2 & 0,7 & 17,6 & 14,3 \\
\hline Halopsis ocellata & 4 & 16 & 55 & 225 & 0,2 & 1,5 & 13,3 & 21,4 \\
\hline Bougainvillia pyramidata* & 0 & 100 & 0 & 1405 & 0,0 & 9,4 & 0,0 & 21,4 \\
\hline Bougainvillia sp. & 0 & 53 & 0 & 748 & 0,0 & 5,0 & 0,0 & 57,1 \\
\hline Aglaura hemistoma & 0 & 9 & 0 & 131 & 0,0 & 0,9 & 0,0 & 14,3 \\
\hline Amphinema rugosum & 0 & 3 & 0 & 36 & 0,0 & 0,2 & 0,0 & 14,3 \\
\hline Aequorea sp. & 0 & 1 & 0 & 7 & 0,0 & 0,0 & 0,0 & 7,1 \\
\hline Modeeria rotunda & 0 & 7 & 0 & 96 & 0,0 & 0,6 & 0,0 & 28,6 \\
\hline
\end{tabular}



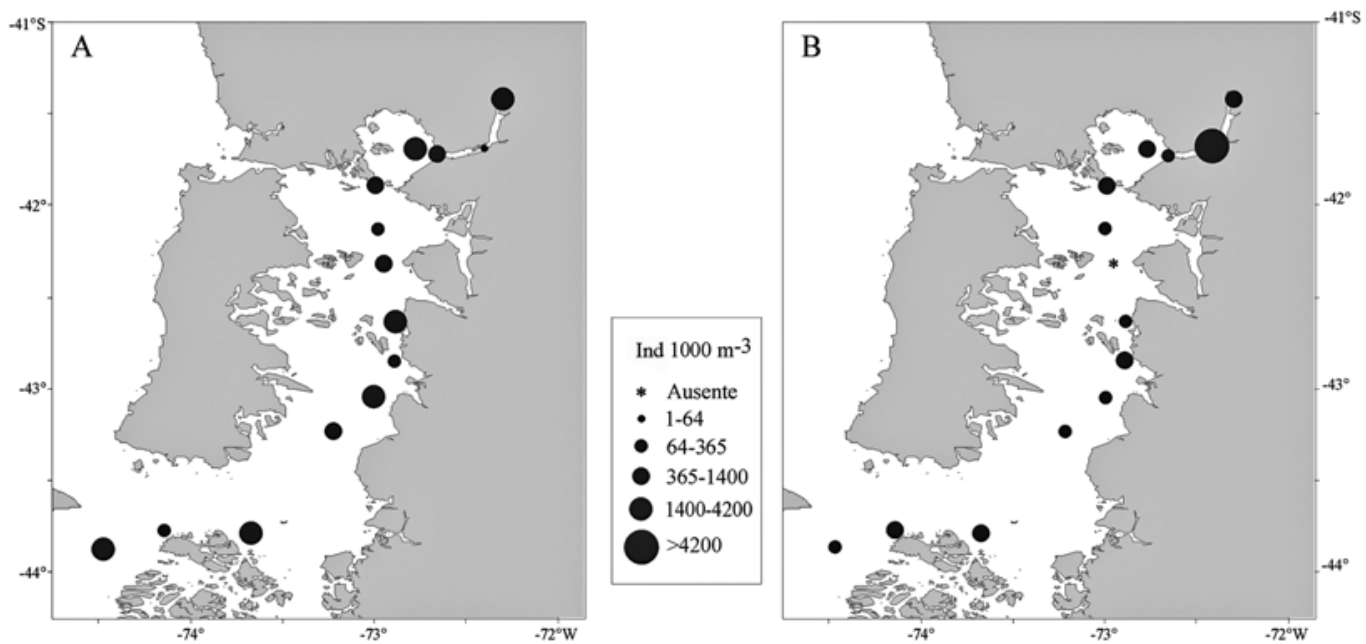

Figura 5

Distribución espacial de Sagitta tasmanica. A) primavera 2004, B) primavera 2005

Spatial distribution of Sagitta tasmanica. A) spring 2004, B) spring 2005
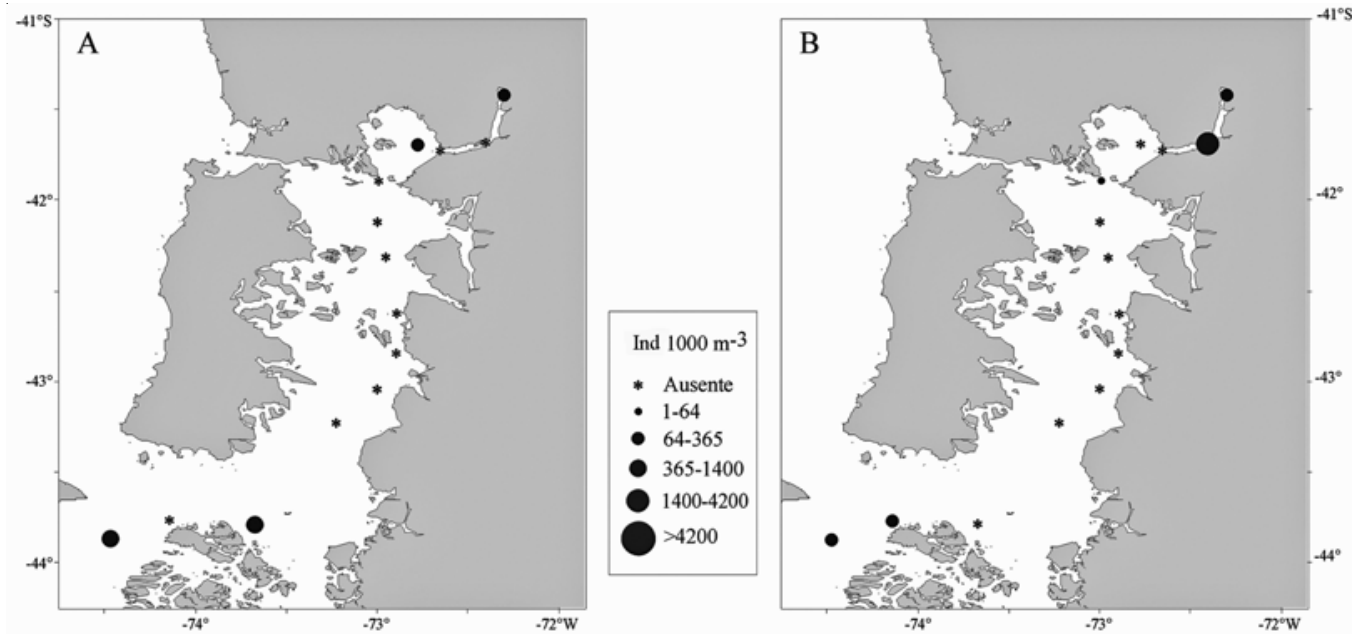

Figura 6

Distribución espacial de Eukrohnia hamata. A) primavera 2004, B) primavera 2005

Spatial distribution of Eukrohnia hamata. A) spring 2004, B) spring 2005

\section{Quetognatos}

Los quetognatos presentaron una amplia distribución latitudinal en ambas primaveras y su abundancia fue 1,8 veces mayor el 2004 respecto del 2005 (Tabla 1). Las mayores agregaciones se determinaron en el fiordo y seno Reloncaví, mientras que en las islas Desertores y la microcuenca sur se obtuvieron los mayores valores de riqueza específica.

Sagitta tasmanica fue la especie predominante y de mayor frecuencia en ambas primaveras. En el 2004 su abundancia promedio fue 2,6 veces superior a la del 2005 (1764 vs. 718 ind. $1000 \mathrm{~m}^{-3}$ respectivamente) (Tabla 1). En ambos periodos los máximos se registraron en el fiordo y seno de Reloncaví, y la disminución registrada el 2005 se observó a lo largo de toda la transecta longitudinal, desde el golfo de Ancud a la Boca del Guafo (Fig. 5).

Eukrohnia hamata fue mucho menos abundante y frecuente que $S$. tasmanica; su abundancia promedio en las primaveras de 2004 y 2005 fue muy similar (147 vs. 

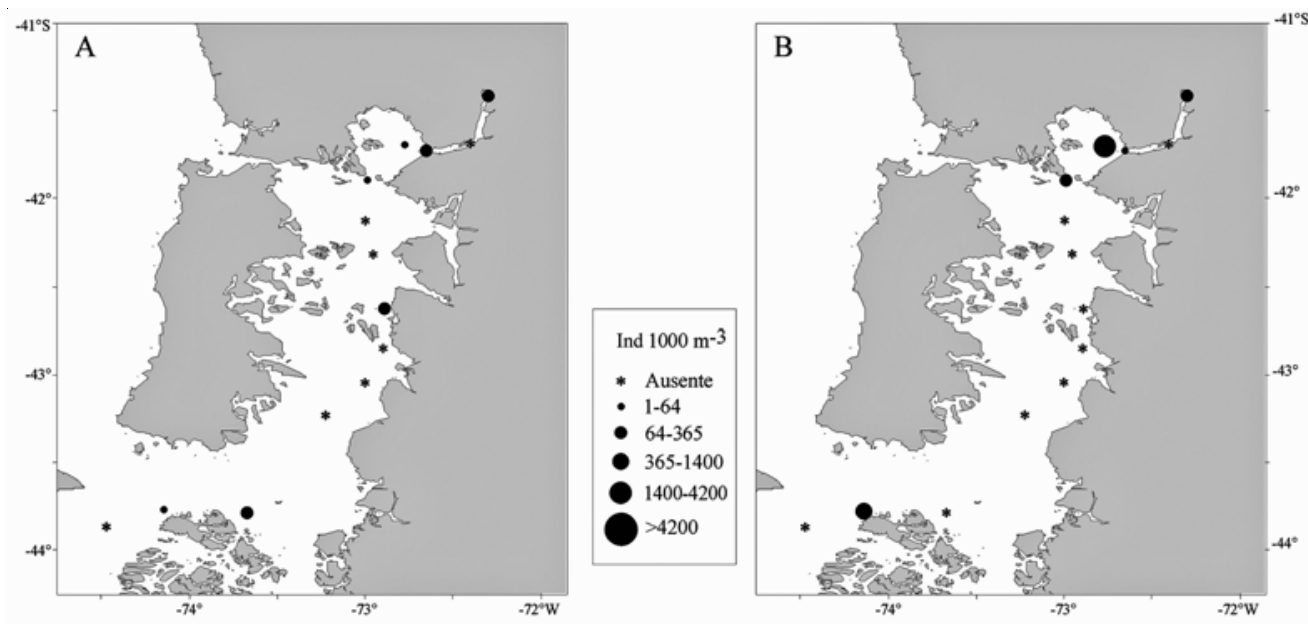

Figura 7

Distribución espacial de Sagitta decipiens. A) primavera 2004, B) primavera 2005

Spatial distribution of Sagitta decipiens. A) spring 2004, B) spring 2005
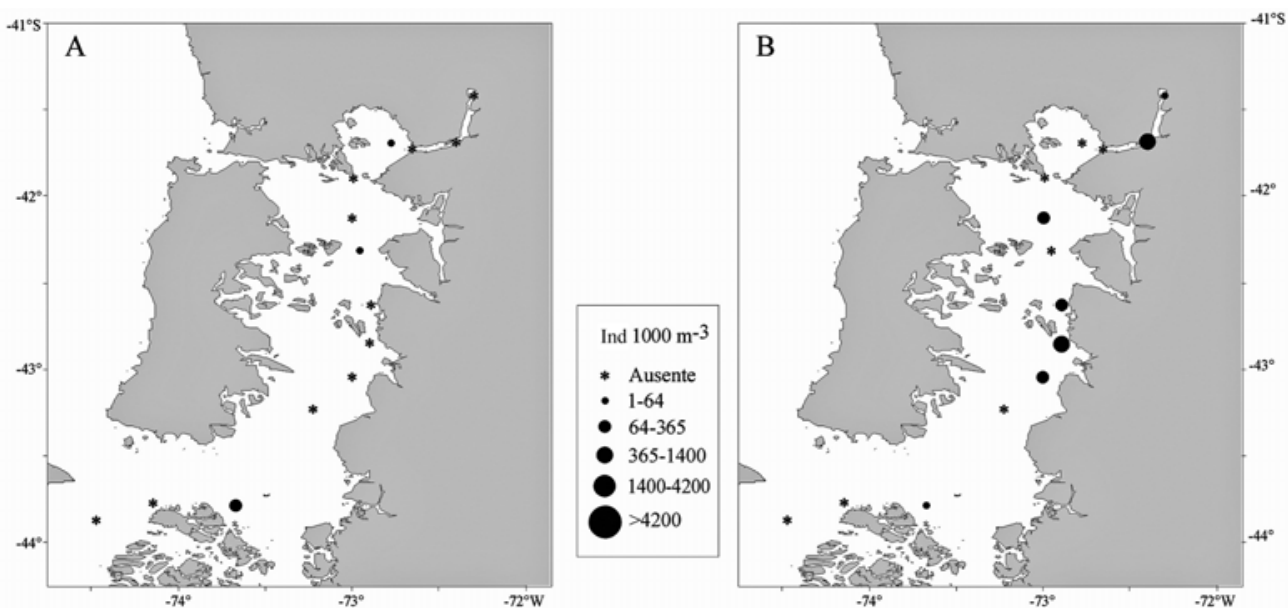

Figura 8

Distribución especial de Sagitta chilensis. A) primavera 2004, B) primavera 2005

Spatial distribution of Sagitta chilensis. A) spring 2004, B) spring 2005

151 ind. $1000 \mathrm{~m}^{-3}$ respectivamente) (Tabla 1). En ambos periodos se encontró exclusivamente en los extremos norte (fiordo y seno Reloncaví) y sur del transecto (Boca del Guafo), y las mayores agregaciones (> 400 ind. 1000 $\mathrm{m}^{-3}$ ) se encontraron en el fiordo Reloncaví en el 2005 (Fig. 6).

Sagitta decipiens incrementó en 3,3 veces su abundancia media entre el 2004 y 2005 (62 vs. 216 ind. $1000 \mathrm{~m}^{-3}$ respectivamente), siendo la segunda especie más abundante después de $S$. tasmanica (Tabla 1). Su distribución geográfica fue similar a la de E. hamata y se recolectó exclusivamente en el fiordo y seno Reloncaví, donde se concentró más del $77 \%$ de los ejemplares, y en la Boca del Guafo (Fig. 7).

Sagitta chilensis fue muy escasa en el 2004, pero su abundancia se incrementó 2,9 veces durante el 2005. A pesar que su distribución geográfica fue muy similar a las dos especies anteriores, en el 2005 presentó algunos núcleos de abundancia en las proximidades de las islas Desertores (Est. 32, 33 y 36) (Fig. 8). 

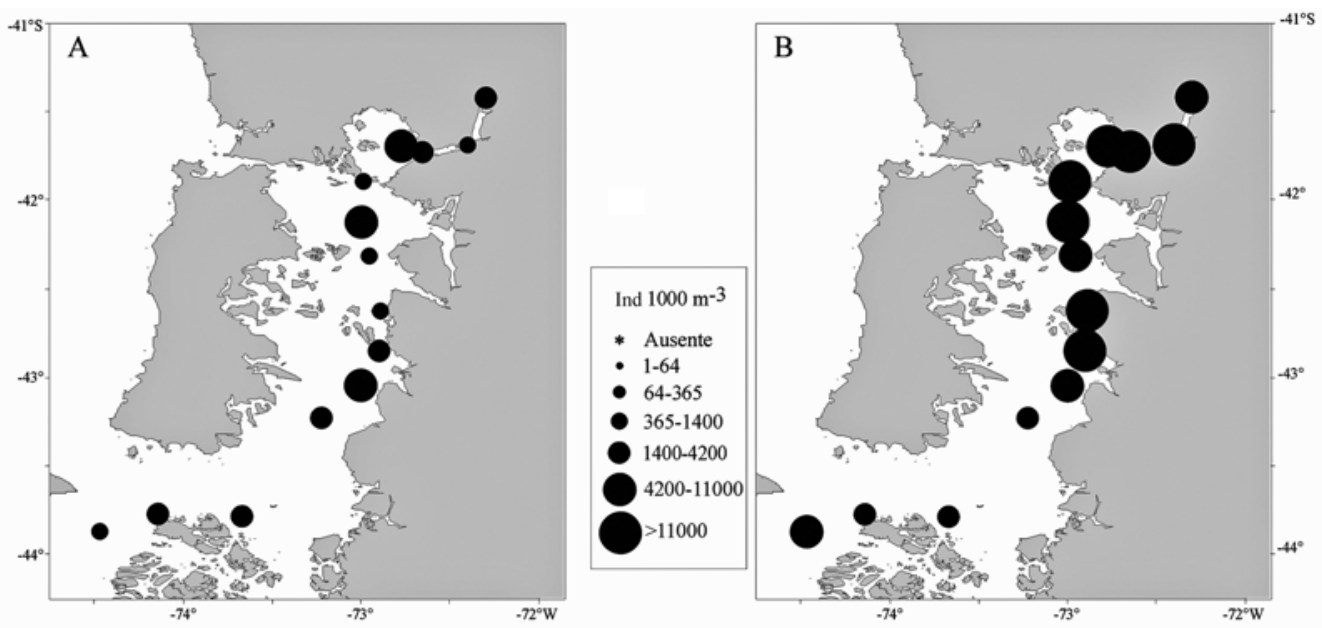

Figura 9

Distribución espacial de Muggiaea atlantica. A) primavera 2004, B) primavera 2005

Spatial distribution of Muggiaea atlantica. A) spring 2004, B) spring 2005
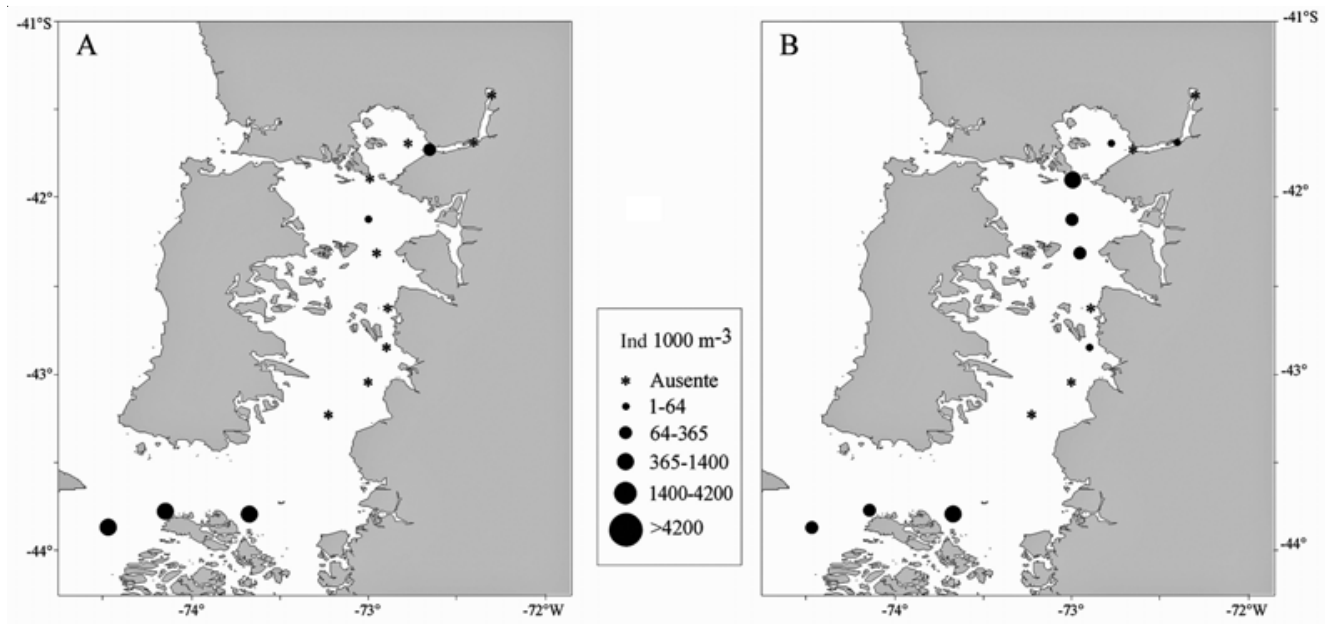

Figura 10

Distribución espacial de Pyrostephos vanhoeffeni. A) primavera 2004, B) primavera 2005

Spatial distribution of Pyrostephos vanhoeffeni. A) spring 2004, B) spring 2005

\section{Sifonóforos}

Los sifonóforos presentaron una amplia distribución latitudinal en ambas primaveras y su abundancia fue 5,1 veces mayor el 2005 con respecto del 2004 (Tabla 1). Se observó una marcada abundancia a lo largo del transecto longitudinal, con una escasa riqueza específica, de solo cinco especies en cada primavera.

Muggiaea atlantica fue la especie predominante y presentó una amplia distribución geográfica, siendo representativa de las fluctuaciones de abundancia de este grupo. Los máximos de abundancia se registraron en la microcuenca norte e islas Desertores y disminuyeron levemente hacia la Boca del Guafo. En primavera de 2005, su abundancia fue 5,6 veces mayor que el 2004, con un máximo de 76.020 ind. $1000 \mathrm{~m}^{-3}$ en el fiordo Reloncaví (Fig. 9).

Pyrostephos vanhoeffeni fue dominante solo en la primavera de 2004, cuando su abundancia fue ligeramente superior (1,3 veces) a la del 2005. En este último año, a pesar de su escasa abundancia $(<1 \%)$ presentó una mayor frecuencia de ocurrencia (64\%) (Tabla 1). En ambas 

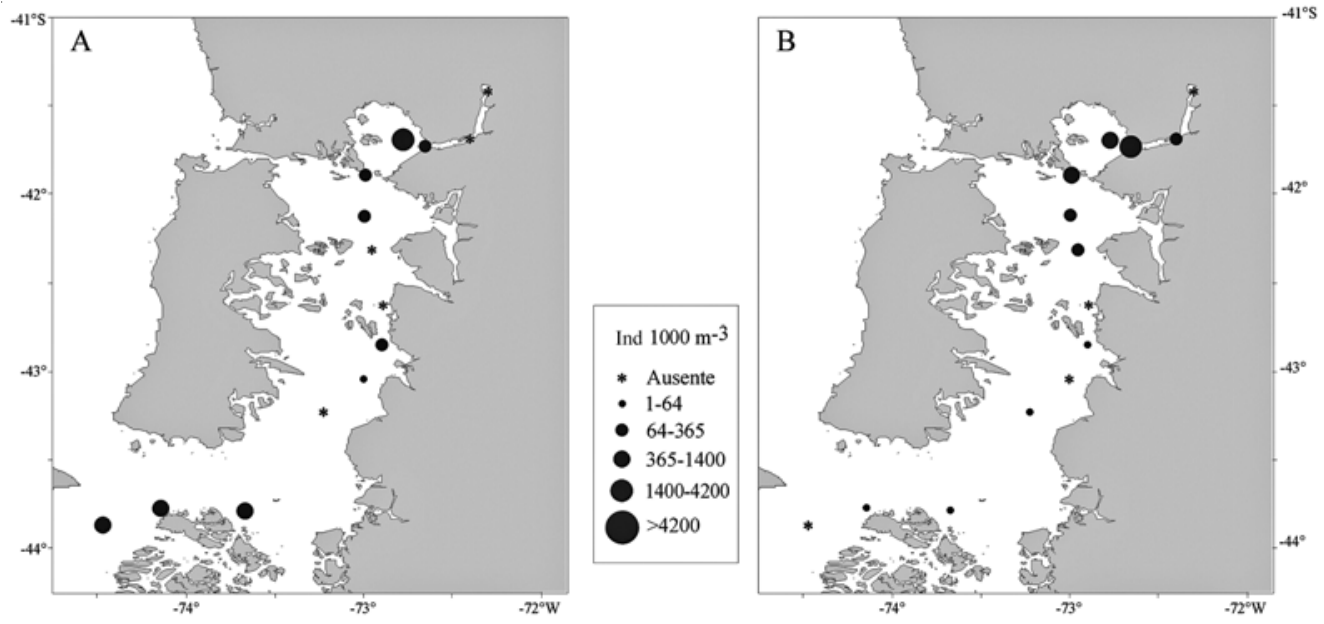

Figura 11

Distribución espacial de Solmundella bitentaculata. A) primavera 2004, B) primavera 2005

Spatial distribution of Solmundella bitentaculata. A) spring 2004, B) spring 2005
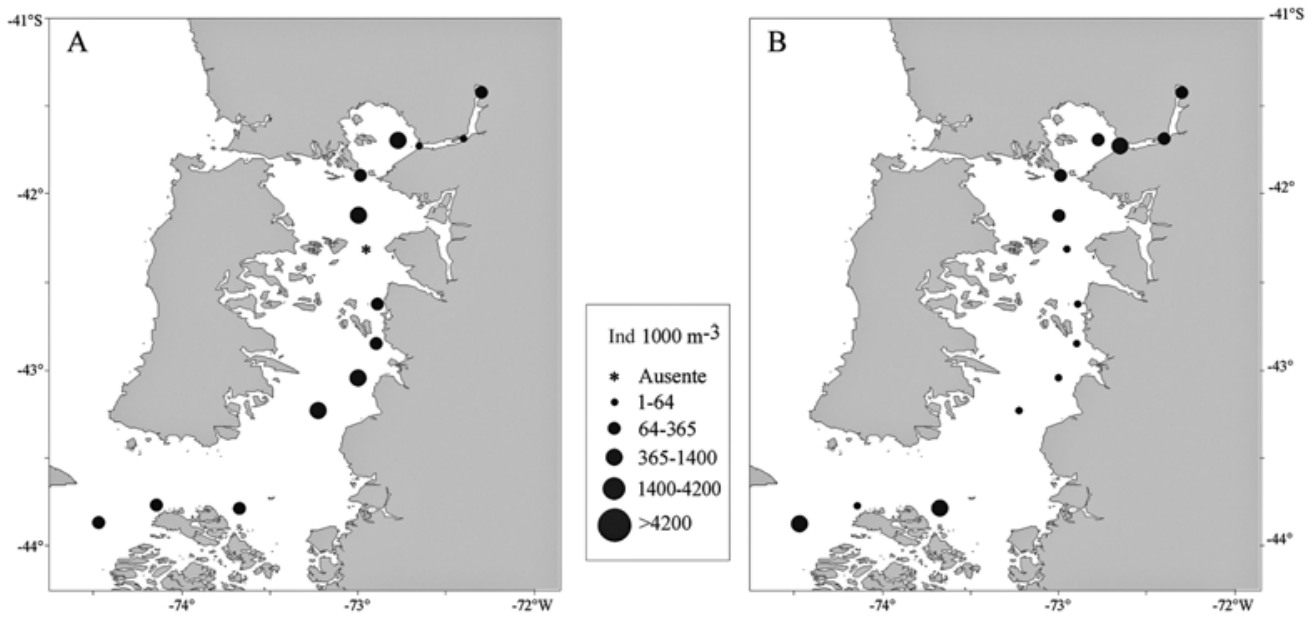

Figura 12

Distribución espacial de Clytia simplex. A) primavera 2004, B) primavera 2005

Spatial distribution of Clytia simplex. A) spring 2004, B) spring 2005

primaveras presentó un patrón similar de distribución y se capturó principalmente en el golfo de Ancud y en la Boca del Guafo, donde alcanzó un máximo de 700 ind. $1000 \mathrm{~m}^{-3}$ (Fig. 10).

\section{Hidromedusas}

Las hidromedusas fueron 1,9 veces más abundantes en la primavera de 2004 respecto de la de 2005. En el 2004 se determinaron altas densidades en todo el transecto oceanográfico, mientras que en el 2005 los ejemplares se concentraron principalmente en el fiordo Reloncaví y en la microcuenca norte. La riqueza específica fue mucho menor el 2004 respecto del 2005 y los mayores valores se obtuvieron en el fiordo Reloncaví, microcuenca norte y Boca del Guafo.

Solmundella bitentaculata fue más abundante en ambos cruceros, con una frecuencia de ocurrencia de 66,7\% el 2004 y de 71,4\% el 2005. En primavera de 2004 los máximos se ubicaron en el seno Reloncaví y Boca del Guafo, mientras que en el 2005 se capturó solamente en la microcuenca norte, con máximos en el seno Reloncaví (Fig. 11). 

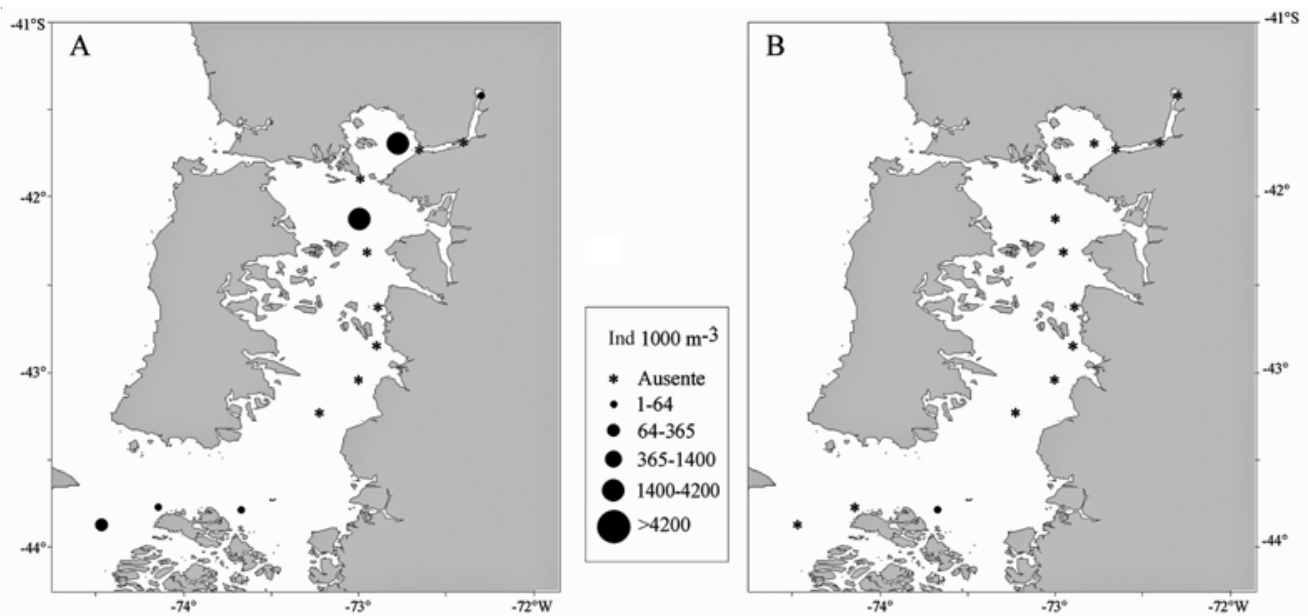

Figura 13

Distribución espacial de Hydractinia tenuis. A) primavera 2004, B) primavera 2005

Spatial distribution of Hydractinia tenuis. A) spring 2004, B) spring 2005
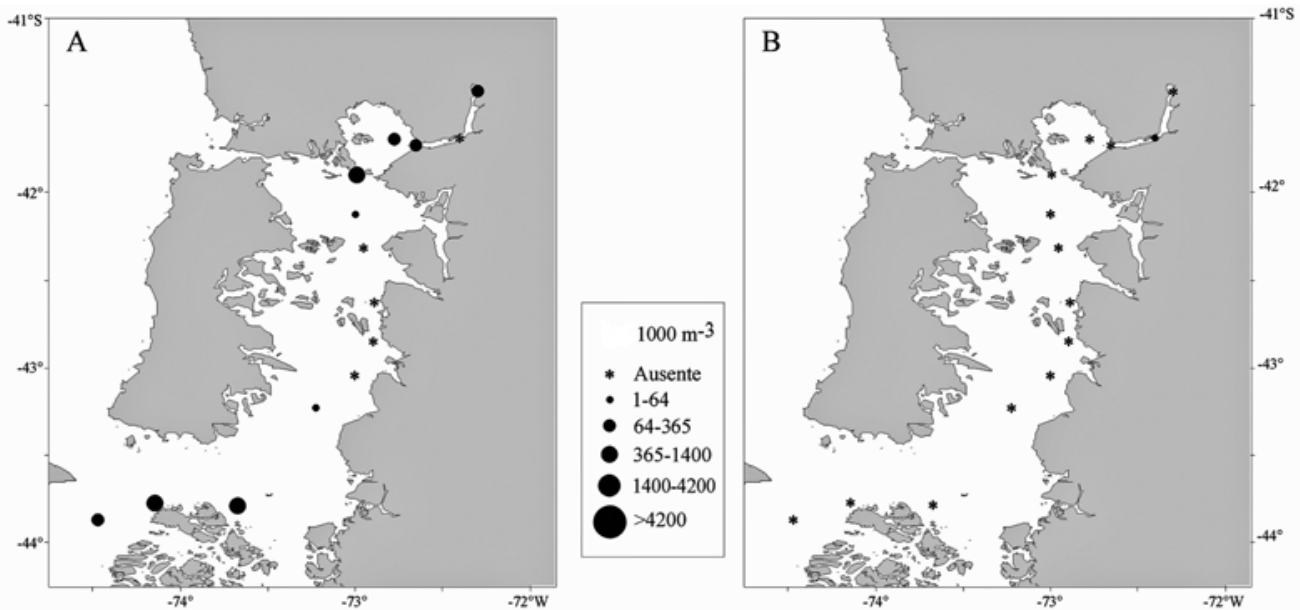

Figura 14

Distribución geográfica de Amphogona apicata. A) primavera 2004, B) primavera 2005

Spatial distribution of Amphogona apicata. A) spring 2004, B) spring 2005

A pesar que Clytia simplex fue menos abundante que S. bitentaculata, presentó una alta frecuencia $(<90 \%)$ en ambas primaveras (Tabla 1). En el 2004 su cantidad fue 2,1 veces mayor que el 2005, alcanzando un máximo en la Boca del Guafo. En cambio, en la primavera de 2005, las mayores densidades se registraron en la microcuenca norte y en la Boca del Guafo (Fig. 12).

Hydractinia tenuis ( $=H$. minuta) y Amphogona apicata fueron dominantes solo en el 2004 (Tabla 1). Los máximos de $H$. tenuis se registraron en el seno Reloncaví y golfo de Ancud y los de A. apicata en la Boca del Guafo. En la primavera de 2005 ambas especies fueron ocasionales (Figs. 13 y 14).

Por otra parte, Bougainvillia muscoides se recolectó en ambos períodos en densas agregaciones al interior del fiordo Reloncaví (Fig. 15), siendo 2,8 veces más abundante en el 2004 respecto del 2005. En el 2004 tuvo una mayor cobertura geográfica, reflejada en una frecuencia de $86,7 \%$, mientras que el 2005, estuvo restringida a la microcuenca norte (Tabla 1 ). 

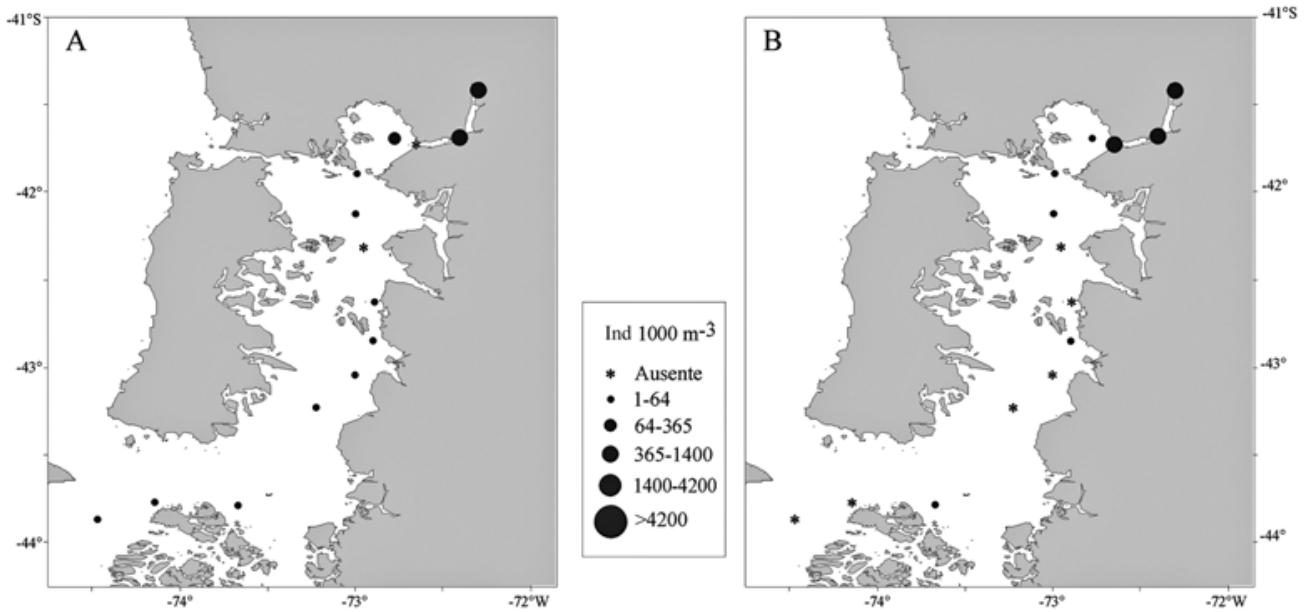

Figura 15

Distribución espacial de Bougainvillia muscoides. A) primavera 2004, B) primavera 2005

Spatial distribution of Bougainvillia muscoides. A) spring 2004, B) spring 2005
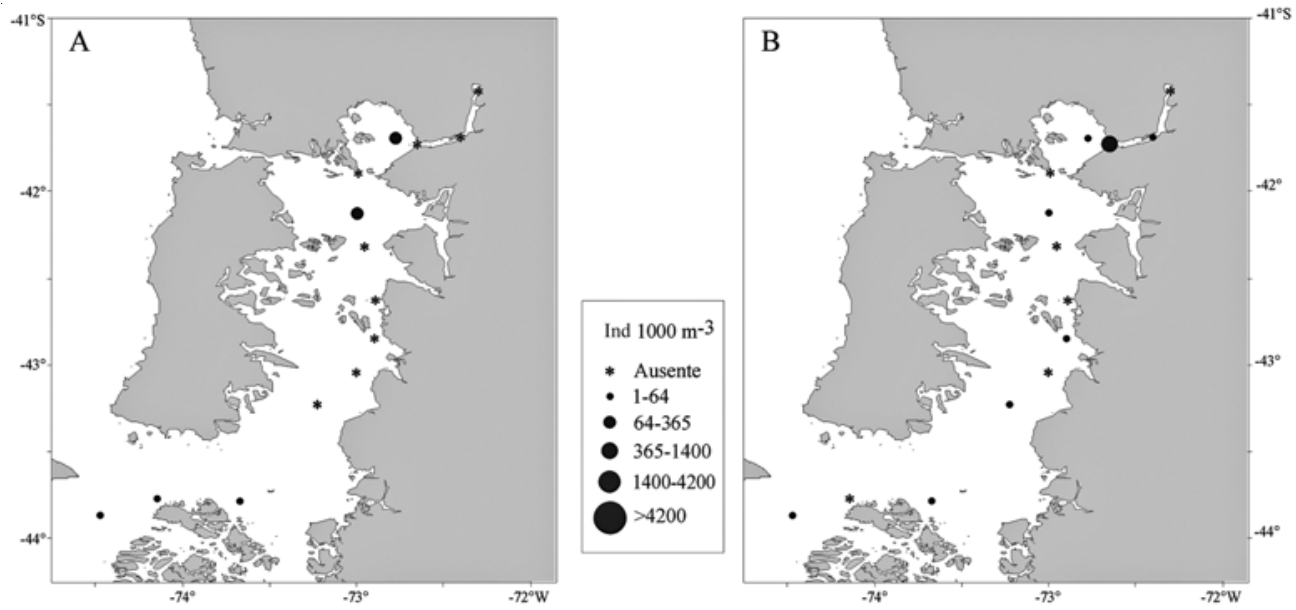

Figura 16

Distribución espacial de Obelia spp. A) primavera 2004, B) primavera 2005

Spatial distribution of Obelia spp. A) spring 2004, B) spring 2005

Por último, Obelia spp. y Cunina peregrina fueron dominantes solo en la primavera de 2005 (Tabla 1), época en que las mayores densidades se recolectaron en el fiordo y seno Reloncaví (Figs. 16 y 17).

\section{Correlación de Pearson y prueba de Mann-Whitney}

Los resultados del índice de correlación de Pearson $(P<$ 0,05) indicaron que Bougainvillia muscoides y Cunina peregrina presentaron una asociación negativa con la temperatura y salinidad, mientras que en Pyrostephos vanhoeffeni esta relación fue negativa con la temperatura y positiva con la salinidad. En cambio, Amphogona apicata, B. muscoides, C. peregrina, Eukrohnia hamata, Sagitta decipiens y $S$. tasmanica presentaron una asociación negativa con la concentración de oxígeno disuelto (Tabla 2). 

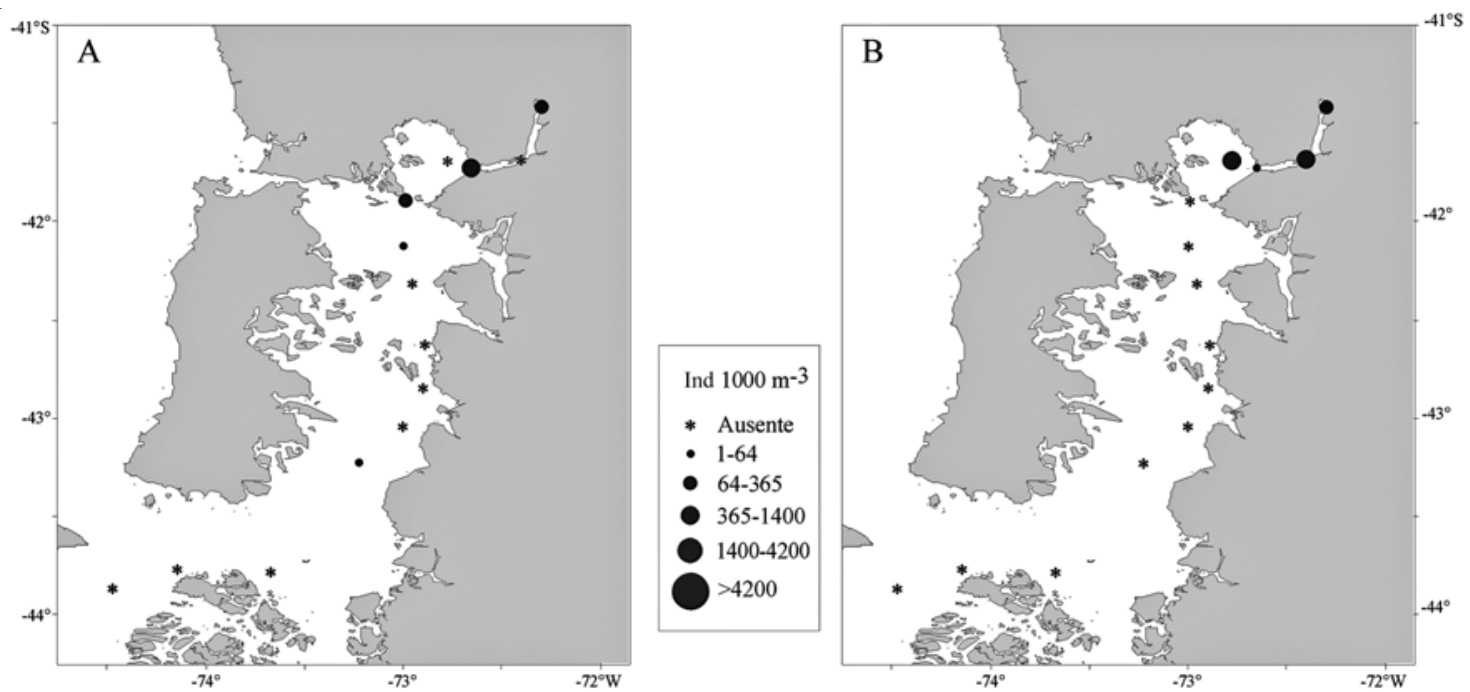

Figura 17

Distribución espacial de Cunina peregrina. A) primavera 2004, B) primavera 2005

Spatial distribution of Cunina peregrina. A) spring 2004, B) spring 2005

\section{Tabla 2}

Correlación de Pearson entre las variables oceanográficas, biomasa quitinosa y gelatinosa, y abundancia de las especies dominantes en las primaveras 2004 y 2005. Los valores significativos se indican en negrita $(\alpha=0,05)$

Pearson correlation between oceanographic variables, chitinous and gelatinous biomass, and abundance of the dominant species in 2004 and 2005 springs. Significant values are indicated in bold $(\alpha=0.05)$

\begin{tabular}{lccc}
\hline \multicolumn{1}{c}{$\begin{array}{c}\text { Variables } \\
\text { bióticas/abióticas }\end{array}$} & $\begin{array}{c}\text { Temperatura } \\
\text { promedio } \\
\left({ }^{\circ} \mathrm{C}\right)\end{array}$ & $\begin{array}{c}\text { Salinidad } \\
\text { promedio }\end{array}$ & $\begin{array}{c}\text { Oxígeno disuelto } \\
\text { promedio } \\
\left(\mathrm{mL} \mathrm{L}^{-1}\right)\end{array}$ \\
\hline Biomasa zooplanctónica & $-0,25$ & 0,24 & $-0,1$ \\
Biomasa gelatinosa & 0,02 & 0,05 & $-0,22$ \\
Amphogona apicata & 0,02 & 0,11 & $\mathbf{- 0 , 4 4}$ \\
Bougainvillia muscoides & 0,47 & $\mathbf{- 0 , 3 9}$ & $\mathbf{- 0 , 5 3}$ \\
Clytia simplex & $-0,03$ & 0,15 & $-0,19$ \\
Cunina peregrina & $\mathbf{- 0 , 4 3}$ & $\mathbf{- 0 , 4 5}$ & $\mathbf{- 0 , 4 5}$ \\
Hydractinia tenuis & $-0,04$ & 0,11 & $-0,26$ \\
Obelia spp. & $-0,07$ & 0,04 & $-0,06$ \\
Solmundella bitentaculata & $-0,02$ & $-0,03$ & $-0,07$ \\
Muggiaea atlantica & 0,11 & $-0,24$ & 0,07 \\
Pyrostephos vanhoeffeni & $\mathbf{- 0 , 5 9}$ & $\mathbf{0 , 4 1}$ & $-0,13$ \\
Eukrohnia hamata & $-0,33$ & 0,27 & $\mathbf{- 0 , 6 6}$ \\
Sagitta decipiens & $-0,07$ & 0,05 & $\mathbf{- 0 , 4 3}$ \\
Sagitta chilensis & $-0,05$ & 0,07 & $-0,09$ \\
Sagitta tasmanica & 0,09 & 0,05 & $\mathbf{- 0 , 4 4}$ \\
\hline
\end{tabular}


Tabla 3

Resultados de la prueba de Mann-Whitney para el total de las especies dominantes. Los valores significativos se indican en negrita $(\alpha=0,05)$

Mann-Whitney test results for the total of dominant species. Significant values are indicated in bold $(\alpha=0.05)$

\begin{tabular}{lrrrc}
\hline \multicolumn{1}{c}{ Especie } & $\mathrm{N}$ & $\mathrm{U}$ & $\mathrm{Z}$ & \multicolumn{1}{c}{$P$} \\
\hline Eukrohnia hamata & 29 & 99 & 0,28368 & 0,745088 \\
Sagitta chilensis & 29 & 80 & $-1,11291$ & 0,202067 \\
Sagitta decipiens & 29 & 99 & 0,26186 & 0,769526 \\
Sagitta tasmanica & 29 & $\mathbf{3 3}$ & $\mathbf{3 , 1 4 2 3 4}$ & $\mathbf{0 , 0 0 1 6 7 6}$ \\
Muggiaea atlantica & 29 & $\mathbf{2 4}$ & $\mathbf{- 3 , 5 3 5 1 3}$ & $\mathbf{0 , 0 0 0 4 0 8}$ \\
Pyrostephos vanhoeffeni & 29 & 92 & $-0,58919$ & 0,531797 \\
Anphogona apicata & 29 & $\mathbf{4 1}$ & $\mathbf{2 , 8 1 5 0 1}$ & $\mathbf{0 , 0 0 1 2 5 5}$ \\
Bougainvillia muscoides & 29 & 68 & 1,61481 & 0,102727 \\
Clytia simplex & 29 & 68 & 1,61481 & 0,106352 \\
Cunina peregrina & 29 & 105 & 0,00000 & 1,000000 \\
Hydractinia tenuis & 29 & $\mathbf{6 8}$ & $\mathbf{1 , 6 3 6 6 3}$ & $\mathbf{0 , 0 2 9 2 4 7}$ \\
Obelia spp. & 29 & 79 & $-1,13473$ & 0,213561 \\
Solmundella bitentaculata & 29 & 91 & 0,61101 & 0,535097 \\
\hline
\end{tabular}

De acuerdo a los resultados de la prueba de MannWhitney para las especies dominantes, se observó que $S$. tasmanica, Muggiaea atlantica, A. apicata e Hydractinia tenuis presentaron diferencias significativas en su abundancia media entre las primaveras de 2004 y 2005. En S. tasmanica, A. apicata y $H$. tenuis la abundancia disminuyó, mientras que en M. atlantica aumentó (Tabla 3).

\section{Discusión}

La distribución de la biomasa de zooplancton quitinoso no mostró una tendencia latitudinal clara y se caracterizó por su alta variabilidad. En el fiordo Reloncaví y la microcuenca norte se determinaron los mayores valores, particularmente en primavera de 2004. Estos resultados confirman estudios anteriores que indican altas biomasas en esta microcuenca, asociadas a su mayor estabilidad oceanográfica (Palma \& Rosales 1997, Palma \& Silva 2004). Además, en el sector norte se determinó la mayor variabilidad interanual, pues en primavera de 2004 los valores fueron un orden de magnitud superior a los de primavera de 2005, como también a los estimados en primavera de 1995 (Palma \& Rosales 1997).

La microcuenca norte, de mayor estabilidad oceanográfica, se caracteriza por altos valores de temperatura y oxígeno disuelto, y bajos valores de salinidad. En cambio, en la microcuenca sur (islas Desertores a Boca del Guafo), se observa un aumento en la salinidad, y una disminución en temperatura y oxígeno disuelto. Este patrón latitudinal parece ser estable en el tiempo y coincide con los resultados obtenidos en esta extensa área en años anteriores (Silva et al. 1995, 1997, Palma \& Silva 2004). Las diferencias entre estas microcuencas se deberían a la presencia del cordón de las islas Desertores que restringe el paso del Agua Subantártica (ASAA) hacia la microcuenca norte.

Al comparar ambas primaveras, se encontró que los valores medios de temperatura registrados el 2004 fueron mayores que el 2005. En consecuencia, las variaciones interanuales de biomasa zooplanctónica podrían ser atribuidas a la disminución de temperatura. Sin embargo, la ausencia de correlación entre la temperatura y la biomasa (Tabla 2), sugiere que estas variaciones podrían ser causadas otros factores, probablemente de carácter trófico.

Los valores de biomasa gelatinosa fueron muy bajos en comparación con los de biomasa quitinosa. A pesar que no presentaron una tendencia latitudinal definida, se observó que los leves incrementos de biomasa gelatinosa estuvieron asociados a descensos en la biomasa quitinosa (Fig. 3). Esta relación inversa se podría atribuir al impacto trófico de algunas especies gelatinosas, impacto que ha sido reportado para numerosas regiones marinas (Fosshagen 1980, Båmstedt et al. 1994, Pàges et al. 2001, Giesecke \& González 2004, Suchman \& Brodeur 2005). 
Este impacto se debería a que los organismos gelatinosos son oportunistas, tienen altas tasas de crecimiento y renovación, y rápidamente pueden formar densas poblaciones, sobre todo en ambientes que presentan algún grado de perturbación (Mills 1995, Kovalev \& Piontkovski 1998, Brodeur et al. 2002). Por ejemplo, se ha señalado que $M$. atlantica, que fue la especie más abundante registrada en este estudio, consume in situ copépodos de 0,1 a 0,9 mm de longitud, con un consumo diario estimado de 5,5 a 10,5 presas por sifonóforo (Purcell 1982). Recientemente, Vargas et al. (2007) estimaron un consumo de $\sim 4$ copépodos diarios por sifonóforo en condiciones experimentales.

Sobre estas proliferaciones, se han propuesto varios factores que explicarían los aumentos poblacionales de organismos gelatinosos en ambientes costeros (CIESM 2001). Entre ellos, se indica que a menudo la eutroficación causa un aumento en la biomasa de cnidarios en desmedro de su diversidad (Arai 2001), mientras que el aumento en la turbidez reduce la visibilidad y puede dar una ventaja competitiva a los depredadores gelatinosos táctiles sobre los depredadores visuales (Eiane et al. 1997, Sørnes \& Aksnes 2004). Sin embargo, no hay estudios que indiquen que las aguas interiores de Chiloé presenten un aumento significativo en la perturbación antropogénica, que sea responsable de las variaciones interanuales en la biomasa gelatinosa. Por lo tanto, es probable que estas variaciones puedan ser causadas por sobrepesca, como ha sido señalado para diversas áreas marinas (Mills 1995, 2001).

A pesar que la riqueza de especies fue menor en primavera de 2004 (27 especies) que en la de 2005 (32 especies), los valores de diversidad fueron mayores el 2004 (0,35-3,03 bits) respecto del 2005 (0,29-2,14 bits) (Fig. 4). Esta aparente inconsistencia fue causada por cambios en la composición y abundancia de algunas especies gelatinosas, como ocurrió con $M$. atlantica, que en el 2005 constituyó densas agregaciones en toda el área de estudio, con promedios de 17.127 ind. $1000 \mathrm{~m}^{-3}$, que afectaron fuertemente la diversidad.

Con respecto a la composición específica se puede señalar que de las 34 especies identificadas, los quetognatos (6) y sifonóforos (6) ya habían sido registrados en esta misma área (Palma \& Rosales 1997, Villenas et al. 2009). En cambio, las 22 especies de hidromedusas identificadas constituyen primeros registros para el área comprendida entre el fiordo de Reloncaví y la Boca del Guafo; 12 de ellas fueron registradas previamente y de manera exclusiva en el fiordo Comau (Galea 2007, Galea et al. 2007) (Tabla 1).
La mayoría de las especies identificadas, especialmente las dominantes, son habitantes comunes en aguas antárticas y subantárticas, y son frecuentes en el ecosistema de canales y fiordos entre Puerto Montt y cabo de Hornos (Palma 2008). La presencia de estas especies en aguas interiores de Chiloé se ve favorecida por el ingreso permanente de aguas del Océano Pacífico adyacente, a través del canal Chacao y Boca del Guafo. Del conjunto de especies dominantes, Sagitta tasmanica, S. chilensis y Pyrostephos vanhoeffeni son endémicas en canales y fiordos australes (Palma 2008); en cambio, Muggiaea atlantica, Solmundella bitentaculata, Clytia simplex, Amphogona apicata, Bougainvillia muscoides, Obelia spp. y Cunina peregrina son de amplia distribución en el Sistema de la Corriente de Humboldt (Fagetti 1973, Palma \& Apablaza 2004), donde incluso algunas como $M$. atlantica, S. bitentaculata, C. simplex y Obelia spp., forman densas agregaciones en áreas costeras (Palma \& Rosales 1995, Ulloa et al. 2000).

Es conocido que las fluctuaciones de temperatura desempeñan un papel preponderante en la composición zooplanctónica, favoreciendo algunas especies sobre otras (Parsons \& Lalli 2002, Kehayias 2004). De hecho, el análisis de Pearson mostró asociaciones significativas entre algunas especies y la temperatura (Tabla 2), como también con respecto a la salinidad, pero este parámetro presentó una escasa variación entre ambos periodos. Por lo tanto, es probable que la disminución de temperatura en primavera de 2005 (Fig. 2), sea responsable de cambios en la abundancia de algunas especies dominantes. En efecto, en primavera de 2005 se observaron incrementos de Sagitta decipiens, S. chilensis, M. atlantica, Obelia spp. y $C$. peregrina, aunque solo en $M$. atlantica fue significativo (Tabla 3). Estos incrementos fueron más relevantes en el sector norte (fiordo Reloncaví a islas Desertores), donde se registró una notoria disminución en la abundancia de varias hidromedusas que son más afines a aguas más cálidas (Tabla 1). Estos cambios de abundancia también podrían ser atribuidos a la competencia interespecífica que ocurre en sistemas altamente productivos (Edwards \& Richardson 2004), que en este caso, habrían favorecido la proliferación explosiva de $M$. atlantica, que causó el aumento de la biomasa gelatinosa y el descenso de la quitinosa en el sector norte, debido a su elevada tasa de predación (Purcell 1982) (Fig. 3b).

Las altas densidades de $M$. atlantica en primavera de 2005, estuvieron asociadas a una significativa disminución en la abundancia de S. tasmanica, H. tenuis y A. apicata (Tabla 3) y adicionalmente, de varias otras hidromedusas (Tabla 1). Se ha observado que la ocurrencia simultánea de dos o más especies en una 
comunidad, refleja un cierto grado de concordancia en los requerimientos ecológicos ambientales o biológicos tendiendo a formar grupos más o menos compactos según la similitud de sus requerimientos (Lie et al. 1983, Gasca et al. 1996). Por lo tanto, es probable que entre $M$. atlantica y estas especies exista cierta concordancia en sus requerimientos ecológicos y consecuentemente sus altas densidades pueden afectar la densidad de las otras. De hecho, estas cuatro especies son dominantes en aguas interiores del ecosistema austral caracterizado por la presencia dominante del Agua Subantártica Modificada y se ha visto en varios sectores que entre ellas no comparten sus áreas de mayor densidad (Palma et al. 2007a, b).

Los resultados obtenidos indican la existencia de cambios interanuales, pero se requiere de una mayor periodicidad en el muestreo, que permita efectuar a lo menos un seguimiento estacional para determinar con mayores fundamentos, los factores ambientales (temperatura, salinidad) o biológicos (disponibilidad trófica, predación), que regulan los cambios registrados en la estructura comunitaria de zooplancton gelatinoso.

\section{Agradecimientos}

Los autores agradecen al Comité Oceanográfico Nacional por el financiamiento de los Proyectos CONA-C10F 0411 (noviembre 2004) y CONA-C11F 05-12 (noviembre 2005), al comandante y a la tripulación del AGOR Vidal Gormaz de la Armada de Chile y a los técnicos de cubierta. Además, se agradece al profesor Nelson Silva de la Escuela de Ciencias del Mar de la Pontificia Universidad Católica de Valparaíso por facilitar los datos oceanográficos.

\section{Literatura citada}

Arai MN. 1988. Interactions of fish and pelagic coelenterates. Canadian Journal of Zoology 9: 1913-1927.

Arai MN. 2001. Pelagic coelenterates and eutrophication: a review. Hydrobiologia 451: 69-87.

Båmstedt U, ME Martinussen \& S Matsakis. 1994. Trophodynamics of the two scyphozoan jellyfishes, Aurelia aurita and Cyanea capillata, in western Norway. ICES Journal of Marine Science 51: 369-382.

Borgel R. 1970/71. Geomorfología de las regiones australes de Chile. Revista Geológica de Chile 21: 135-140.

Brodeur R, H Sugisaki \& G Hunt. 2002. Increases in jellyfish biomass in the Bering Sea: implications for the ecosystem. Marine Ecology Progress Series 233: 89-103.

CIESM. 2001. Gelatinous zooplankton outbreaks: theory and practice. Commission Internationale pour l'Exploration Scientifique de la Mer Méditerranée, Monaco. CIESM Workshop Series 14: 1-112.
Chapin FS III, ES Zavaleta, VT Eviner, RL Naylor, PM Vitousek, HL Reynolds, DU Hooper, S Lavorel, OE Sala, SE Hobbie, MC Mack \& S Díaz. 2000. Consequences of changing biotic diversity. Nature 405: 234-242.

Edwards M \& AJ Richardson. 2004. Impact of climate change on marine pelagic phenology and trophic mismatch. Nature 430: 881-884.

Eiane K, DL Aksnes \& J Giske. 1997. The significance of optical properties in competition among visual and tactile predators: a theoretical study. Ecological Modelling 98: 123-136.

Fagetti E. 1973. Medusas de aguas chilenas. Revista de Biología Marina 15(1): 31-75.

Fosshagen A. 1980. How the zooplankton community may vary within a single fjord system. En: Freeland HJ, DV Farmer \& CD Levings (eds). Fjord oceanography, pp. 399-405. Plenum Publishing, New York.

Galea HR. 2007. Hydroids and hydromedusae (Cnidaria: Hydrozoa) from the fjords region of southern Chile. Zootaxa 1597: 1-116.

Galea HR, V Häussermann \& G Försterra. 2007. Hydrozoa, fjord Comau, Chile. Check List 3(2): 159-167.

Gasca R, JN Alvarez-Cadena \& E Suárez-Morales. 1996. Chaetognath assemblages in the Mexican Caribbean Sea (1991). Caribbean Marine Studies 5: 41-50.

Giesecke R \& HE González. 2004. Feeding of Sagitta enflata and vertical distribution of chaetognaths in relation to low oxygen concentrations. Journal of Plankton Research 26: 475-486.

Jackson JB, MX Kirby, WH Berger, KA Bjorndal, LW Botsford, BJ Bourque, RH Bradbury, R Cooke, J Erlandson, JA Estes, TP Hughes, S Kidwell, CB Lange, HS Lenihan, JM Pandol, CH Peterson, RS Steneck, MJ Tegner \& RR Warner. 2001. Historical overfishing and the recent collapse of coastal ecosystems. Science 293: 629638.

Kehayias G. 2004. Spatial and temporal abundance distribution of chaetognaths in eastern Mediterranean pelagic waters. Bulletin of Marine Science 74(2): 253-270.

Kidwell S. 2007. Discordance between living and death assemblages as evidence for anthropogenic ecological change. Proceedings of the National Academy of Sciences of the United States of America 104(45): 17701-17706.

Kovalev AV \& SA Piontkovski. 1998. Interannual changes in the biomass of the Black Sea gelatinous zooplankton. Journal of Plankton Research 20: 1377-1385.

Lie U, T Magnesen, B Tunberg \& D Aksnes. 1983. Preliminary studies on the vertical distribution of sizefractions in the zooplankton community in Lindaspollene, western Norway. Sarsia 68: 65-80.

Mianzan HW \& RA Guerrero. 2000. Environmental patterns and biomass distribution of gelatinous macrozooplankton. Three study cases in the South-western Atlantic Ocean. Scientia Marina 64(1): 215-224. 
Mills C. 1995. Medusae, siphonophores, and ctenophores as planktivorous predators in changing global ecosystems. ICES Journal of Marine Science 52: 575-581.

Mills C. 2001. Jellyfish blooms: are populations increasing globally response to changing ocean conditions? Hydrobiologia 451: 55-68.

Pagès F, H González, M Ramón, M Sobarzo \& JM Gili. 2001. Gelatinous zooplankton assemblages associated with water masses in the Humboldt Current System and potential predatory impact by Bassia bassensis (Siphonophora: Calycophorae). Marine Ecology Progress Series 210: 1324

Palma S. 2008. Zooplankton distribution and abundance in the austral Chilean channels and fjords. En: Silva N \& S Palma (eds). Progress in the oceanographic knowledge of Chilean interior waters from Puerto Montt to Cape Horn, pp. 107-113. Comité Oceanográfico Nacional-Pontificia Universidad Católica de Valparaíso, Valparaíso.

Palma S \& P Apablaza. 2004. Abundancia estacional y distribución vertical del zooplancton gelatinoso carnívoro en un área de surgencia en el norte del Sistema de la Corriente de Humboldt. Investigaciones Marinas 32(1): 4970.

Palma S \& S Rosales. 1995. Composición, abundancia y distribución estacional del macroplancton de la bahía de Valparaíso. Investigaciones Marinas 23: 49-66.

Palma S \& S Rosales. 1997. Sifonóforos epipelágicos de los canales australes de Chile $\left(41^{\circ} 30^{\prime}-46^{\circ} 40^{\prime}\right.$ 'S). Ciencia y Tecnología del Mar 20: 125-146.

Palma S \& N Silva. 2004. Distribution of siphonophores, chaetognaths and euphausiids and oceanographic conditions in the fjords and channels of southern Chile. Deep-Sea Research II 51(6-9): 513-535.

Palma S, P Apablaza \& N Silva. 2007a. Hydromedusae (Cnidaria) of the Chilean southern channels (from the Corcovado Gulf to the Pulluche-Chacabuco Channels). Scientia Marina 71(1): 65-74.

Palma S, P Apablaza \& D Soto. 2007b. Diversity and aggregation areas of planktonic cnidarians of the southern channels of Chile (Boca del Guafo to Pulluche Channel). Investigaciones Marinas 35(2): 71-82.

Parsons TR \& CM Lalli. 2002. Jellyfish populations explosions: revisiting a hypothesis of possible causes. La Mer 40: 111-121.
Purcell JE. 1982. Feeding and growth of the siphonophore Muggiaea atlantica (Cunningham, 1893). Journal of Experimental Marine Biology and Ecology 62: 39-54.

Ramírez B \& E Pizarro. 2005. Distribución de clorofila a y feopigmentos en los canales australes chilenos comprendidos entre Puerto Montt y la laguna San Rafael, Chile. Ciencia y Tecnología del Mar 28(1): 45-62.

Silva N, H Sievers \& R Prado. 1995. Descripción oceanográfica de los canales australes de Chile. Zona Puerto

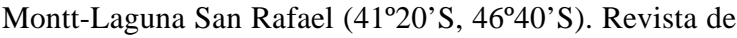
Biología Marina 30(2): 207-254.

Silva N, C Calvete \& H Sievers. 1997. Características oceanográficas físicas y químicas de canales australes chilenos entre Puerto Montt y Laguna San Rafael (Crucero CIMAR-Fiordo 1). Ciencia y Tecnología del Mar 20: 23106.

Sokal R \& FJ Rohlf. 1995. Biometry: the principles and practice of statistics in biological research, $832 \mathrm{pp}$. W.H. Freeman and Company, New York.

Sørnes TA \& DL Aksnes. 2004. Predation efficiency in visual and tactile planktivores. Limnology and Oceanography 49: 69-75.

Suchman CL \& RD Brodeur. 2005. Abundance and distribution of large medusae in surface waters of the northern California Current. Deep-Sea Research II 52: 5172.

Ulloa R, S Palma, L Linacre \& N Silva. 2000. Seasonal changes in the bathymetric distribution of siphonophores, chaetognaths and euphausiids associated to water masses off of Valparaiso, Chile (Southeast Pacific). En: Farber J (ed). Oceanography of the Eastern Pacific 1: 72-83. Centro de Investigación Científica y de Educación Superior de Ensenada (CICESE), Ensenada.

Vargas CA, RA Martínez, LA Cuevas, MA Pavez, C Cartes, HE González, R. Escribano \& G. Daneri. 2007. The relative importance of microbial and classical food webs in a highly productive coastal upwelling area. Limnology and Oceanography 52(4): 1495-1510.

Villenas F, S Palma \& D Soto. 2009. Chaetognath spatial and how egg-carrying affects the vertical distribution of Sagitta tasmanica off southern Chile. Scientia Marina 73(1): 2938. 